\title{
Repeated Resection for Pulmonary Metastatic Hepatocellular Carcinoma after Liver Transplantation- Case Reports and a Proposal of Negative Predictors for the Recurrence after the Operation
}

\author{
Tomoyuki Nakagiri*, Soichiro Funaki, Yasushi Shintani, Masayoshi Inoue, Noriyoshi Sawabata, \\ Masato Minami, Meinoshin Okumura
}

Department of General Thoracic Surgery, Graduate School of Medicine, Osaka University, Osaka, Japan.

Email: *gilly64@thoracic.med.osaka-u.ac.jp

Received October $4^{\text {th }}$, 2012; revised November $5^{\text {th }}$, 2012; accepted November $12^{\text {th }}, 2012$

\begin{abstract}
Background: Orthotropic liver transplantation (OLTx) is recognized as a radical therapy for unresectable hepatocellular carcinoma (HCC) without distant metastasis. Although the outcome depends on distant recurrence of HCC, the predictors for recurrence after repeat resection are unknown. Case 1: A 52-year-old man, who had suffered from hepatitis $\mathrm{B}$ and underwent repeat local ablation therapies since 50 years old, underwent living-donor OLTx because of multiple HCC recurrence with tumor marker (TM) elevation but without distant metastasis. Histopathological diagnosis was moderately differentiated HCC. After the operation, he got TM normalization and was managed with cyclosporine A, without rejection. Although he underwent adjuvant chemotherapy, a pulmonary metastasis was found 1 year after the OLTx. He underwent wedge resection of the lung using video-assisted thoracoscopic surgery (VATS). Half a year after the operation, a recurrence was found in the transplanted liver with TM elevation. While a local ablation therapy was performed, TM was not normalized and new recurrence was found at the hilum of the right lung. Right upper sleeve lobectomy was performed, but he developed multiple recurrences, and died 4 months after the last operation. Case 2: A 32-year-old man, who has suffered from multiple HCC with hepatitis B and underwent hepatic resection and local ablation therapies since 28 years old, underwent living-donor OLTx because of multiple HCC recurrence without distant metastasis. Histopathological diagnosis was moderately differentiated HCC. He was managed using tacrolimus without rejection. Three years after the OLTx, a pulmonary recurrence was found without TM elevation. He underwent wedge resection using VATS. Four year after the last operation, a small recurrence was identified in the right lung without TM elevation, again. Wedge resection using VATS was performed. At the final follow-up visit, 3 years after the last operation, the patient was disease free with normal TM level. Comments: The long survivor without re-recurrence matched only few factors with negative predictors for recurrence after OLTx for HCC, while the other case had almost all factors present. The predictors may be useful also for the patients of the repeat pulmonary metastasectomy after OLTx for HCC.
\end{abstract}

Keywords: Hepatocellular Carcinoma; Liver Transplantation; Recurrence; Lung

\section{Introduction}

Orthotopic liver transplantation (OLTx) is a radical therapeutic option for patients with unresectable nonmetastatic hepatocellular carcinoma (HCC) [1,2]. However, tumor recurrence is still a major limitation of long-term survival [1], with the most common site of distant recurrence being the lung [3]. Although cases of solitary pulmonary resection for HCC recurrence following OLTx have been reported [1], negative predictors of re-recurrence after

\footnotetext{
"Corresponding author.
}

repeat resection for pulmonary metastasis is still unknown. We present 2 cases of repeated pulmonary resections for metastatic HCC following OLTx and discuss the recurrence predictors of repeat pulmonary metastasectomy after OLTx along with consideration of a previously reported case.

\section{Case Reports}

\subsection{Case 1}

A 54-year-old man was admitted to our hospital for HCC 
pulmonary metastasis following OLTx. At the age of 50, he had been diagnosed with hepatitis $\mathrm{B}$, and suffered from multiple HCC recurrence with elevated tumor markers [serum alpha-fetoprotein (AFP) $128 \mathrm{ng} / \mathrm{ml}$ (nor$\mathrm{mal}<20 \mathrm{ng} / \mathrm{ml}$ ), PIVKA II level $877 \mathrm{mAU} / \mathrm{ml}$ (normal $<$ $40 \mathrm{mAU} / \mathrm{ml})]$. He underwent percutaneous ethanol injection therapy (PEIT), transcatheter arterial embolization (TAE), and radiofrequency ablation (RFA) for the lesions in S3, S5, S6, S7 and S8. After these therapies, tumor markers were normalized. At the age of 52, computed tomography (CT) showed bilobar multiple new small lesions, sized about $1.5 \mathrm{~cm}$ in each, along with elevated AFP level $(510 \mathrm{ng} / \mathrm{ml})$, without distant metastasis, again.

For unresectable multiple HCC recurrence without metastasis, he underwent living-donor OLTx from his son. The histopathological diagnosis was moderately differentiated HCC with capsule and micro-vascular invasions. After the OLTx, his AFP level became normal. He was managed with prednisolone and cyclosporine A, without rejection. In addition, He underwent adjuvant chemotherapy with doxorubicin and 5FU, and interferon therapy.

Another year after the OLTx, chest CT scanning revealed an 8-mm solid lesion in the right lung along with an elevated AFP level (Figure 1(A)). He underwent interferon therapy and chemotherapy with tegafur/uracil (UFT). However, the tumor size was increased, and AFP level was elevated to $275 \mathrm{ng} / \mathrm{ml}$. He underwent a wedge resection under video-assisted thoracoscopic surgery (VATS) for the pulmonary lesion. After the operation, AFP level was decreased, but was not normalized (70 $\mathrm{ng} / \mathrm{ml})$. Five months after the metastasectomy, routine follow-up CT scanning revealed solitary intrahepatic recurrence, $2 \mathrm{~cm}$ in size, with elevated AFP level. This lesion was ablated with TAE, though his AFP level remained high (492 ng/ml).

Another year after the last TAE, a 2-cm solitary recurrence lesion was revealed in the right lung near the second carina without lymph node swelling. He underwent a right sleeve upper lobectomy and mediastinal lymph node sampling, considering primary lung cancer (Figure 1(B)). Histopathological findings revealed HCC recurrence and metastasis in the level \#4 lymph nodes. Unfortunately, 2 months after the operation, he developed multiple pulmonary and intrahepatic metastases, and died 4 months after the last operation.

\subsection{Case 2}

A 35-year-old man who underwent OLTx for HCC was admitted to our hospital to undergo a pulmonary metastasectomy. He had suffered from multiple HCC tumors

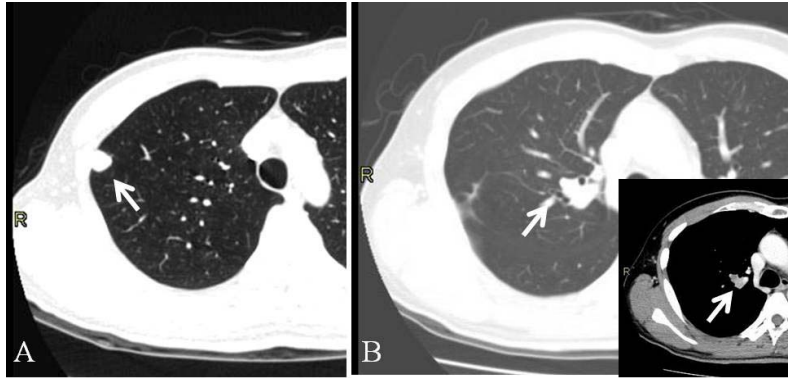

Figure 1. Metastatic lesions of hepatocellular carcinoma in the lungs of case 1 (arrows). (A) A right upper lobe (S1) nodule was resected with wedge resection under VATS; (B) A right upper lobe (S1) nodule was also resected with sleeve lobectomy. The small image shows the difference between tumor and vessels with contrast image.

along with hepatitis B, and underwent an S4 segmentectomy and S6 microwave coagulation therapy (MCT) of the liver at the age of 28 . Another year after the operation, he had HCC recurrence and underwent an S3 segmentectomy, and S5 and S8 MCT, after which he was given a subcutaneously embedded reservoir for hepatic arterial chemotherapy. Even after arterial chemotherapy, HCC locally recurred in S1 and S5 of the liver without elevation of AFP level. For multiple recurrence of HCC without metastasis, he underwent living-donor OLTx from his father at the age of 32, and was managed with prednisolone and tacrolimus without rejection. The histological diagnosis was moderate differentiated HCC with micro-vascular invasions.

Three years after the OLTx, a 1-cm coin lesion appeared in the left lung on a follow-up CT scan image without tumor marker elevations (Figure 2(A)). For diagnosis, wedge resection under VATS was performed. The lesion was diagnosed as metastasis of HCC.

Four years after the operation, follow-up CT revealed a 6-mm coin lesion in the right lung without tumor marker elevation (Figure 2(B)). The lesion then gradually enlarged over the next 2 months. Partial resection of the lung under VATS was performed, and this lesion was also diagnosed as HCC metastasis. At the final follow-up visit (10.8 years after OLTx, 3 years after second lung resection), the patient was disease free with normal tumor markers.

\section{Discussion}

A lung metastasectomy is recognized as a curative treatment strategy for patients with lung metastasis from several types of cancer, when the original site is controlled. Previous studies have reported that lung metastasectomy for HCC metastasis is also effective and a few have shown prolongation of survival with repeat resection for HCC pulmonary metastasis [4]. Then, considering a case 


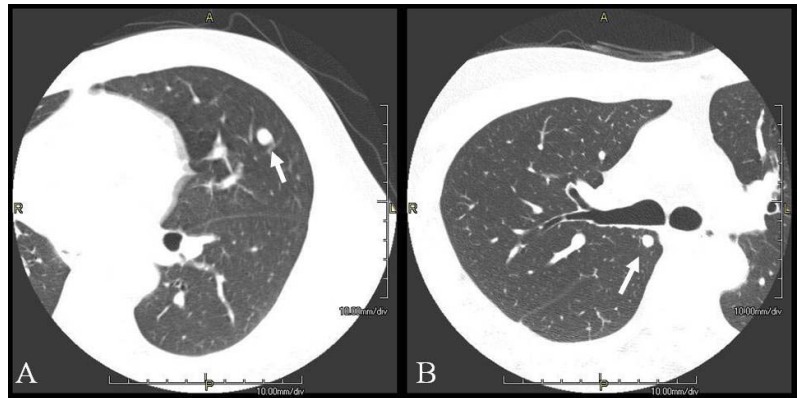

Figure 2. Metastatic lesions of hepatocellular carcinoma in the lungs of case 2 (arrows). (A) A left upper lobe (S5) nodule was resected with wedge resection under VATS; (B) A right upper lobe (S2) nodule was also resected with wedge resection under VATS.

after OLTx for HCC, recurrence is also the rate-limiting factor for long term survival in approximately $20 \%$ of patients [5].

Zimmerman et al. published a review of HCC recurrence following OLTx, in which lymph node metastasis, micro- and macro-vascular invasion, tumor size larger than $5 \mathrm{~cm}$, tumor grade, bilobar disease, total number of lesions, elevated AFP level, and earlier recurrence $(<1$ year) were shown to be significant negative predictors [1]. Kornberg et al. reported that application of an immunosuppression strategy with cyclosporine A (vs. tacrolimus) was also a negative predictor [2].

Previously, only Bazan HA has been reported a long surviving case of repeat pulmonary metastasectomy after OLTx in detail, [5] who suffered from micro-vascular invasion, and multiple and bilobar lesions, whereas no lymph node metastasis, small size well-differentiated HCC at the time of the OLTx. After OLTx, the case was managed with tacrolimus, and had no significant elevation of serum AFP level while the recurrences. The disease-free survival was for 2 years after transplantation and no recurrence in the transplanted liver (Table 1).

Our first patient, who died 3 years after transplantation, had a small sized and moderately differentiated HCC without lymph node metastasis at the time of OLTx. However, he suffered from micro-vascular invasion, multiple and bilobar lesions, elevated AFP level, and early recurrence after OLTx. In addition, intrahepatic recurrence was found. Before the second metastasectomy, we saw no evidence of lymph node metastasis and planned a lobectomy instead of a sleeve lobectomy of the lung. However, a sleeve lobectomy was eventually performed, because of the tumor location noted during surgery. In addition, he was treated with cyclosporine for immunosuppression (Table 1).

The other patient, who has been alive more than 10 years after transplantation, also suffered from HCC with micro-vascular invasion, and multiple and bilobar lesions, whereas no lymph node metastasis, small size, and moderately differentiated HCC. The HCC characteristics were similar compared with the 1st case, though no significant elevation of AFP was seen in the second. In addition, that patient had disease-free survival for 3 years after transplantation and no recurrence in the transplanted liver. The metastasectomy were performed as wedge resections of the lung, while tacrolimus was used as the immunosuppressive drug (Table 1).

Comparing these cases based on the recurrence after 2nd metastasectomy, case 1 was older than case 2 and had differences in the number of lesions and serum AFP level at OLTx. After OLTx, the case was managed with Cyclosporin A, and the others were managed with tacrolimus (FK506). Disease free periods of case 1 were shorter than those of the others. In addition, serum AFP level of the case 1 rose, while those of the others did not rise.

The age difference might affect the immunity for the recurrence. However, previous reports about pulmonary metastasis from HCC insisted that there was no significant difference between young patients and old patients on the survival or pulmonary metastasis free survival [6-8]. The number of lesions could also affect to the results. Zimmerman described one of the strict criteria that were used to select patients for OLT was no more than 3 tumor nodules [1]. The case 1 had 5 HCC lesions at the time of OLTx. It was clearly contra indication on the criteria. About immunosuppressive therapy, SiamakpourReihani and colleagues have showed that tacrolimus has a potential of malignant tumor growth inhibiter. The potential might affect the result of outcome also to our patients. In addition, the therapy for the recurrence lesion might affect the outcomes. A previous report presented that there was no significant difference among lung procedures [9]. However, lobectomy damages the patient's immunity more than wedge resection [10]. In addition, our case 1 had undergone chemotherapy. These therapies could affect the outcomes.

In a comparison of our cases with their patient in regard to the aforementioned 9 factors, only a few were associated with the 2 long-term survivors, while the our case 1 had 8 factors present. The negative predictors may be useful as the recurrence predictors also for the patients of the repeat pulmonary metastasectomy after OLTx. To decide the indication of repeat pulmonary metastasectomy after OLTx for HCC, the predictors should be also considered.

\section{Conclusion}

Two cases of repeat pulmonary resection for HCC metastases after OLTx were presented. In addition, the negative predictors for recurrence after OLTx for HCC 
Table 1. Patient characteristic in previous and present cases.

\begin{tabular}{|c|c|c|c|}
\hline & Previous case $^{*}$ & Case 1 & Case 2 \\
\hline $\operatorname{Age}^{2 *} /$ Gender & 62/Male & 52/Male & 32/Male \\
\hline Infection state & Hepatitis B & Hepatitis B & Hepatitis B \\
\hline \multicolumn{4}{|l|}{ Primary site } \\
\hline Lymph node metastasis & No & No & No \\
\hline Vascular invasion & Micro-invasion & Micro-invasion & Micro-invasion \\
\hline Tumor size (Max) & $4.0 \mathrm{~cm}$ & $2.0 \mathrm{~cm}$ & $1.5 \mathrm{~cm}$ \\
\hline Tumor grade & Well diff. ${ }^{3^{*}}$ & Mod. diff. ${ }^{4^{*}}$ & Mod. diff. \\
\hline Laterality & Bilateral & Bilateral & Bilateral \\
\hline Number of lesions & 2 & 5 & 2 \\
\hline$A F P^{5^{*}}$ level & Normal & $510 \mathrm{ng} / \mathrm{ml}$ & Normal \\
\hline \multicolumn{4}{|l|}{ After $\mathrm{OLT}^{6{ }^{*}}$} \\
\hline Immunosuppression & FK506 & Cyclosporin A & FK506 \\
\hline Adjuvant therapy & No & $\mathrm{ChT}^{7^{*}}$ and $\mathrm{IFNT}^{8^{*}}$ & No \\
\hline Disease-free period & 2 years & 1 year & 3 years \\
\hline \multicolumn{4}{|l|}{ First recurrence } \\
\hline Tumor size & $<1 \mathrm{~cm}$ & $1 \mathrm{~cm}$ & $1 \mathrm{~cm}$ \\
\hline Laterality & Bilobar & Right & Left \\
\hline AFP level & Normal & $275 \mathrm{ng} / \mathrm{ml}$ & Normal \\
\hline Therapy & Wedge resection & Wedge resection + ChT & Wedge resection \\
\hline \multicolumn{4}{|l|}{ Second recurrence } \\
\hline Disease-free period & 2 years & 1 year & 4 years \\
\hline Tumor size & Not described & $2 \mathrm{~cm}$ & $6 \mathrm{~mm}$ \\
\hline Laterality & Bilateral & Right & Right \\
\hline AFP level & Normal & $492 \mathrm{ng} / \mathrm{ml}$ & Normal \\
\hline Therapy & Wedge resection & Sleeve lobectomy & Wedge resection \\
\hline Outcome (after OLTx) & Alive $\left(6 \mathrm{y}^{9 *}\right)$ & Dead (2.3 y) & Alive (10.8 y) \\
\hline
\end{tabular}

Italic: Proposal indication criteria. ${ }^{*}$ Bazan HA (reference 3 ); ${ }^{2 *}$ At time of transplantation; ${ }^{3 *}$ Well differentiated hepatocellular carcinoma; ${ }^{4 *}$ Moderately differentiated hepatocellular carcinoma; ${ }^{5 *}$ Serum alpha-fetoprotein level; ${ }^{6 *}$ Orthotopic liver transplantation; ${ }^{7 *}$ Chemotherapy; ${ }^{8^{*}}$ Interferon therapy; ${ }^{9 *}$ Years.

may be useful as the predictors also for the patients of the repeat pulmonary metastasectomy after OLTx for HCC.

\section{Limitations}

Our proposal is based on the results of only 3 limited cases. We can only propose the recurrent predictors. We hope this report promotes additional studies to consider this area.

\section{REFERENCES}

[1] M. A. Zimmerman, R. M. Ghobrial, M. J. Tong, et al., "Recurrence of Hepatocellular Carcinoma Following Liver Transplantation: A Review of Preoperative and Postop- erative Prognostic Indicators,” Archives of Surgery, Vol. 143, No. 2, 2008, pp. 182-188. doi:10.1001/archsurg.2007.39

[2] A. Kornberg, B. Küpper, A. Tannapfel, et al., "Long-Term Survival after Recurrent Hepatocellular Carcinoma in Liver Transplant Patients: Clinical Patterns and Outcome Variables,” European Journal of Surgical Oncology, Vol. 36, No. 3, 2010, pp. 275-280. doi:10.1016/j.ejso.2009.10.001

[3] M. J. Bates, E. Farkas, D. Taylor, et al., "Pulmonary Resection of Metastatic Hepatocellular Carcinoma after Liver Transplantation," The Annals of Thoracic Surgery, Vol. 85, No. 2, 2008, pp. 412-415. doi:10.1016/j.athoracsur.2007.10.065

[4] M. Aramaki, K. Kawano, A. Sasaki, et al., "Prolonged Survival after Repeat Resection of Pulmonary Metastasis 
from Hepatocellular Carcinoma," Journal of HepatoBiliary-Pancreatic Surgery, Vol. 9, No. 3, 2002, pp. 386388. doi:10.1007/s005340200046

[5] H. A. Bazan, K. A. Mamurtry, P. F. Waters and S. N. Thung, "Surgical Resection of Pulmonary Metastases after Orthotopic Liver Transplantation for Hepatocellular Carcinoma," Transplantation, Vol. 73, No. 6, 2002, pp. 1007-1009. doi:10.1097/00007890-200203270-00034

[6] F. Chen, K. Sato, T. Fujinaga, et al., "Pulmonary Resection for Metastases from Hepatocellular Carcinoma,” World Journal of Surgery, Vol. 32, No. 10, 2008, pp. 2213-2217. doi:10.1007/s00268-008-9684-8

[7] J. B. Kwon, K. Park, Y. D. Kim, et al., "Clinical Outcome after Pulmonary Metastasectomy from Primary Hepatocellular Carcinoma: Analysis of Prognostic Factors,” World Journal of Gastroenterology, Vol. 14, No. 37, 2008, pp.
5717-5722. doi:10.3748/wjg.14.5717

[8] Y. S. Yoon, H. K. Kim, J. Kim, et al., "Long-Term Survival and Prognostic Factors after Pulmonary Metastasectomy in Hepatocellular Carcinoma,” Annals of Surgical Oncology, Vol. 17, No. 10, 2010, pp. 2795-2801. doi:10.1245/s10434-010-1073-5

[9] M. Kawamura, J. Nakajima, H. Matsuguma, et al., "Surgical Outcomes for Pulmonary Metastases from Hepatocellular Carcinoma,” European Journal Cardio-Thoracic Surgery, Vol. 34, No. 1, 2008, pp. 196-199. doi:10.1016/j.ejcts.2008.03.056

[10] W. Jungraithmayr, C. Frings, G. Zissel, et al., "Inflammatory Markers in Exhaled Breath Condensate Following Lung Resection for Bronchial Carcinoma,” Respirology, Vol. 13, No. 7, 2008, pp. 1022-1027. 